\section{Sensitivity analysis of weight reduction results of an obser- vational cohort study in over- weight and obese children and adolescents in Germany: the EvAKuJ Study}

\author{
Thomas Böhler, 1 Cornelia Goldapp,2 \\ Reinhard Mann,2 Thomas Reinehr,3 \\ Monika Bullinger, ${ }^{4}$ Reinhard Holl, 5 \\ Ulrike Hoffmeister, 5 Andreas van \\ Egmond-Fröhlich, 6 \\ Ulrike Ravens-Sieberer, ${ }^{7}$ Nora Wille ${ }^{7}$ \\ Joachim Westenhöfer, 8 Jürgen Bengel 9 \\ 1 Medical Service of Statutory Health \\ Insurance Baden-Württemberg,

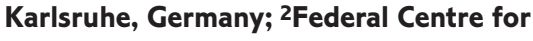 \\ Health Education, Köln, Germany; \\ 3Vestische Kinder- und Jugendklinik, \\ University of Witten-Herdecke, Datteln, \\ Germany; ${ }^{4}$ Department of Medical \\ Psychology, University Clinic Hamburg- \\ Eppendorf, Hamburg, Germany; 5 Institute \\ of Epidemiology and Medical Biometry, \\ University of Ulm, Germany; 6SMZ Ost, \\ Department of Pediatrics, Donauspital, \\ Vienna, Austria; ${ }^{7}$ Research Section Child \\ Public Health, Department of \\ Psychosomatics in Children and \\ Adolescents, University Clinic Hamburg- \\ Eppendorf, Hamburg, Germany; \\ 8Department for Health Sciences, \\ University of Applied Sciences, Hamburg, \\ Germany; ${ }^{\text {Department }}$ of Rehabilitation \\ Psychology and Psychotherapy, Institute \\ of Psychology, University of Freiburg, \\ Germany
}

\section{Abstract}

In the German EvAKuJ observational cohort study, changes in the body mass index standard deviation score (BMI-SDS) of overweight and obese children and adolescents as primary outcome of multimodal (short, inpatient or long, outpatient) weight-loss interventions are difficult to interpret. Published intention-totreat (ITT) and per protocol data obtained at the end of the intervention $\left(\mathrm{T}_{1}\right)$, one year $\left(\mathrm{T}_{2}\right)$, and two years $\left(\mathrm{T}_{3}\right)$ after its end were used for sensitivity analysis of treatment success rates. The odds ratio and the number needed to treat (NNT) for BMI-SDS reduction of at least -0.2 (successful treatment) and at least -0.5 (good treatment success) were related to spontaneous BMI-SDS reduction rates in a hypothetical control group (control event rate, CER). At
$\mathrm{T}_{1}$, treatment seems to be effective up to a CER of $10 \%$ in inpatients and of $5 \%$ in outpatients. ITT analysis, compromised by a loss to followup of 81 to $90 \%$ (inpatients) and 57 to $66 \%$ (outpatients), indicated that treatment may become less effective at a CER above 1\% in inpatients (e.g., successful treatment at $\mathrm{T}_{2}$ : $\mathrm{NNT}=106$, at $\mathrm{T}_{3}: \mathrm{NNT}=51$ ), and above $5 \%$ in outpatients (successful treatment at $\mathrm{T}_{2}$ : $\mathrm{NNT}=7$, at $\mathrm{T}_{3}$ : NNT $=8$; good treatment success at $\mathrm{T}_{2}$ and $\mathrm{T}_{3}$ : NNT=25). Positive short-term effects of inpatient treatment of overweight and obese children and adolescents may not be maintained in the long term. Long-term effectiveness of outpatient treatment may depend on age and the degree of overweight.

\section{Introduction}

According to the German KiGGS study, $18.7 \%$ of children and adolescents with an age between 3 and 17 years are overweight, and an additional $6.3 \%$ are obese. Thus, approximately 1.1 million overweight and 800,000 obese children and adolescents are actually living in Germany. Several different interventions of obesity treatment and secondary and tertiary prevention of obesity-related diseases are routinely applied to these patients. ${ }^{2}$ In recent years several studies on elements of care of overweight and obese children and adolescents have been performed and results of these trials have recently been summarised.3,4 Despite the considerable differences found in the level of available external evidence between these elements, the reviews recommend multimodal interventions which include parents and members of the family or the community (such as schoolmates, teachers and caterers in schools). The interventions should simultaneously address aspects of nutrition (including food quality) and physical activity (aiming at a change from a sedentary to a more active lifestyle including sports). To reach these aims, the interventions should use behavior management principles and techniques and rely upon parents and school teachers as agents of change. ${ }^{5}$ Before the intervention begins, motivation of the child, adolescent, and the family should be assessed and eventually enhanced.

In Germany, inpatient treatment of 4 to 6 weeks' duration is offered in pediatric rehabilitation clinics and is financed by the German Pension Funds (Deutsche Rentenversicherung) and by the German Statutory Health Insurance Funds (Gesetzliche Krankenversicherung, GKV). Allocation to inpatient treatment of overweight and obese children and adolescents follows criteria based on the bio-psychosocial model of overweight and obesity in
Correspondence: Thomas Böhler, MDK BadenWürttemberg - BBZ Karlsruhe, Steinhäuserstrasse 7a, D-76135 Karlsruhe, Germany. Tel. +49.0721.91261.4500 - Fax: +49.0721.91261.4554. E-mail: thomas.boehler@mdkbw.de

Key words: patient education, rehabilitation, body-mass-index, STROBE statement.

Acknowledgments: the EvAKuJ Study was funded by the BZgA. Its results were obtained by the members of the study group in collaboration with providers of treatment programs listed in the Appendix (Study Group and Treatment Centers). Interim results of the study have been presented in yearly intervals at the meeting of the German Obesity Society (Deutsche AdipositasGesellschaft) and were published as shown in the Appendix (Publications of the Study Group). A glossary lists definitions of frequently used terms and abbreviations in the Appendix. Components of the interventions for inpatients and outpatients are shown in the Appendix.

Contributions: the authors contributed equally.

Conflicts of interests: TB has been involved in accreditation of providers of ambulatory patient education and training programs for obese children and adolescents by German Statutory Health Care Funds. CG and RM were involved in designing the EvAKuJ study and provided its financial frame; they were advised by TB and JB. TR, AvEF and JW offered inpatient or outpatient weightreduction programs for children and adolescents.

Received for publication: 9 June 2013.

Accepted for publication: 30 September 2013.

This work is licensed under a Creative Commons Attribution NonCommercial 3.0 License (CC BYNC 3.0).

@C Copyright T. Böhler et al., 2013

Licensee PAGEPress, Italy

Pediatric Reports 2013; 5:e16

doi:10.4081/pr.2013.e16

youth. 6 The GKV additionally offer structured patient education and training programs of 6 to 12 months duration as part of outpatient treatment for overweight and obese children. ${ }^{7}$ Access to such a program is usually granted according to a national consensus statement of representatives of GKV, health care providers, and federal health authorities in the following circumstances: 8 i) for extremely obese patients with a BMI above the $99.5^{\text {th }}$ percentile; ii) for obese patients with a BMI above the $97^{\text {th }}$ and up to the $99.5^{\text {th }}$ percentile, if additional risk factors (insulin resistance, hypercholesterolemia, family disposition for diabetes mellitus type 2, myocardial infarction, or stroke) or illnesses related to increased body weight (arterial hypertension, diabetes mellitus type 
2, disturbed glucose tolerance, premature onset of puberty, polycystic ovary syndrome, orthopedic symptoms) are involved; iii) for overweight patients with a BMI above the $90^{\text {th }}$ and up to the $97^{\text {th }}$ percentile with one or more of the previously listed illnesses if they require specific treatment.

The Federal Centre of Health Education in Germany (Bundeszentrale für gesundheitliche Aufklärung, BZgA) had calculated in 2004 and 2005 that each year an estimated number of 44,000 children, adolescents, and their caregivers participated in more than 700 intervention programs for inpatients and/or outpatients. ${ }^{2}$ These programs are complex health interventions, ${ }^{9}$ whose long-term effectiveness has not yet been convincingly demonstrated. 3,4 In 2005, the BZgA therefore initiated a project for evaluation of obesity treatment in children and adolescents in Germany (Evaluation der Adipositastherapie bei Kindern und Jugendlichen in Deutschland; EvAKuJ). The study project was aiming at longitudinal observation of a cohort of overweight and obese children and adolescents receiving therapeutic interventions by randomly selected providers (see Appendix for the complete list of publications). 10 The study was planned and organized by the EvAKuJ Study Group (Appendix), who performed analysis and published reports of all available data in yearly intervals. The study was solicited, financed, registered, and monitored by the BZgA. Study protocol and results as well as contact data of the participating health care providers (Appendix) had been made available to the public (http://www.bzgakinderuebergewicht.de).

The study recruited patients from July 2005 until September 2006 with the intention to follow them up for 2 years thereafter. The baseline characteristics of the study cohort have been extensively described (Appendix) and outcome data at the end of the intervention (Appendix) and at least one year after the completion of the intervention (Appendix) have been published by the EvAKuJ study group. ${ }^{10}$ Concentrating on the primary outcome variable, we present a detailed analysis of shortterm, mid-term and long-term changes of bodymass-index (BMI) standard deviation scores (SDS) from baseline until approximately two years after the end of the intervention, in the inpatient and the outpatient treatment setting. Sensitivity analysis of the data collected by the EvAKuJ Study group allowed us to present these results according to criteria of evidencebased reporting of observational studies and to discuss them using a model for implementation of complex health interventions that has recently been refined. ${ }^{9-14}$

\section{Materials and Methods}

The EvAKuJ study was conducted by a multidisciplinary research team (EvAKuJ Study Group, Appendix) as an observational multicenter cohort study. Out of 477 providers of multimodal weight reduction interventions, who had in part been identified during a preceding study and who were contacted by the research team, ${ }^{2} 135$ declared their interest to participate in the study and to recruit patients without any financial benefit or other incentives. Of these, 52 providers were chosen by chance. These providers were classified as belonging to one out of six clusters of treatment (see glossary in the Appendix). It was initially estimated that at least 300 patients had to be recruited into interventions in each cluster. Thus, a sample size of 1800 was assumed to be sufficient for assessment of treatment success rates and their comparative analysis between clusters.

Each provider independently included patients into the study if they were between 8 and 16 years old and had a BMI above the $90^{\text {th }}$ percentile for sex and age at baseline. 15 Furthermore, at least data from one of the psychometric questionnaires (Appendix) assessing aspects of health-related quality of

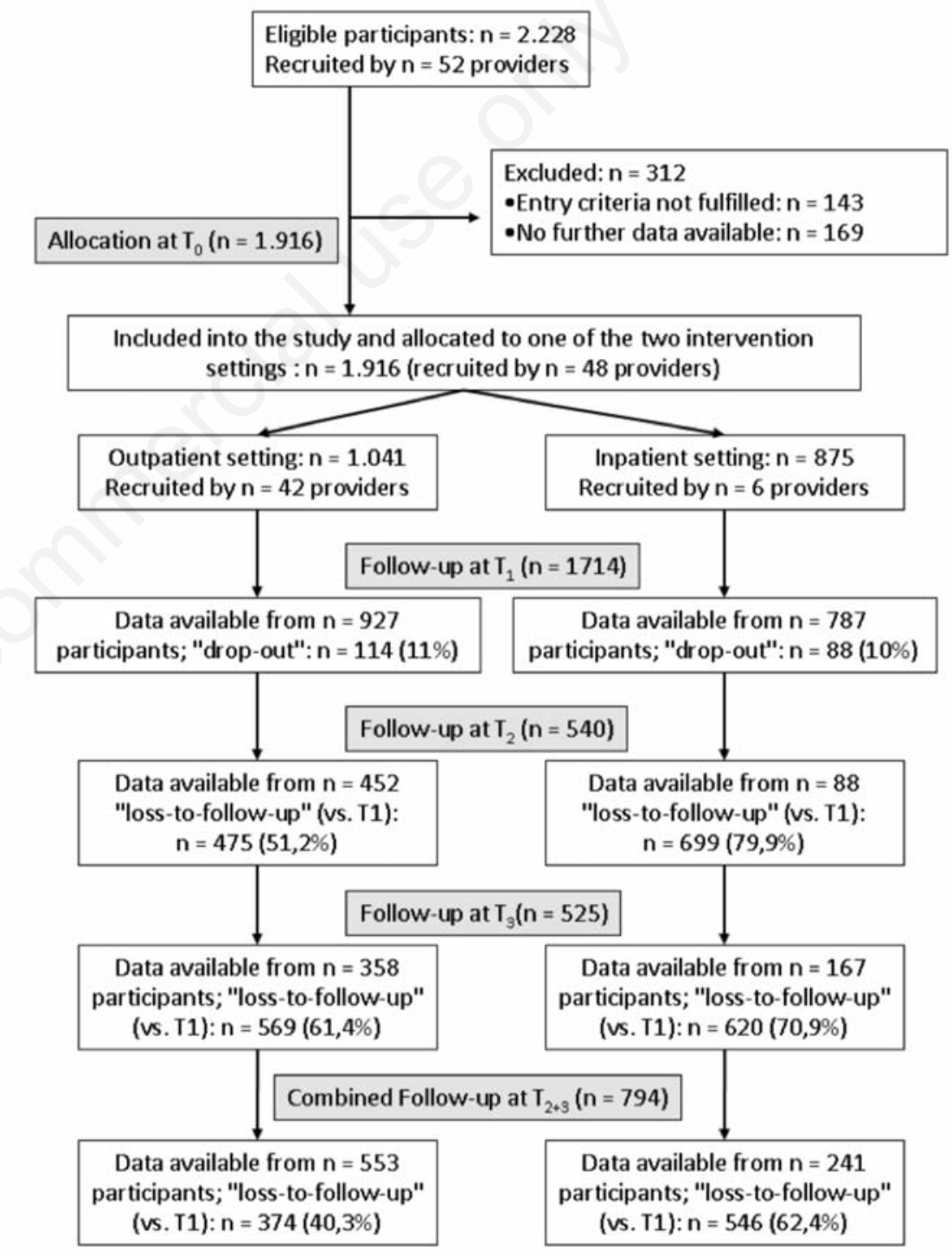

Figure 1. Flow diagram showing patient recruitment and follow-up in the EvAKuJ study. Data on combined follow-up at any time point after the end of the intervention $\left(T_{2+3}\right)$ are not reported here but were used in a previous publication of the EvAKuJ Study Group (Appendix). 
life, eating behavior and food frequency, physical behavior and leisure activity - had to be provided at baseline, i.e. before entering the study. At baseline (time point $\mathrm{T}_{0}$ ), at the end of the intervention (time point $\mathrm{T}_{1}$ ), and approximately one year after the end (time point $\mathrm{T}_{2}$ ) and two years after the end (time point $\mathrm{T}_{3}$ ), each patient's actual body height and weight were either measured or obtained from files together with information on blood pressure, blood glucose, and lipid status. In addition, psychometric questionnaires had to be completed as described (Appendix).

Recruitment was oriented at 300 patients per cluster and was open for all clusters between July 2005 and September 2006. However, the estimated sample size of 300 patients was reached only in two clusters. In retrospect, the short half-life of providers of weight-loss interventions for children and adolescents in Germany was identified as one potential cause of this observation: each year, approximately $10 \%$ of the providers included in the assessment of the BZgA in 2004 and 2005 had ceased their activities mostly due to economic reasons (Appendix).2,10 Thus, comparative cluster analysis was not possible. Therefore, during post hoc analysis, those 48 providers who actively participated in the study were allocated to either the inpatient or the outpatient treatment setting, using the duration of the intervention as denominator. A duration of less than 3 months (short therapy) indicated inpatient treatment in a rehabilitation clinic, while duration of more than 3 months (long therapy) corresponded to outpatient treatment usually by a multidisciplinary team.

The timeline of the study and the typical interventions and processes in both treatment settings are described in the Appendix. Since data on structures and processes in the different treatment centers were lacking, in the Appendix we used a published blueprint for standard treatment schemes in order to describe the two treatment modalities which were compared post hoc. ${ }^{6}$ According to Perera et al., 16 components of complex interventions are regarded either as objects of fixed nature (Appendix, squared brackets), or as activities with a certain flexibility (represented by parentheses). As further specified in the Appendix, a behavioral weight control program corresponding to current national guidelines was offered in both settings. ${ }^{17}$ The general aims were to reduce energy intake, diminish sedentary behavior, and increase physical activity of overweight and obese children and adolescents. Intervention methods included behavior modification techniques such as selfmonitoring with food and activity diaries, goal setting, stimulus control and positive reinforcement. In the outpatient setting, children, adolescents, and their families were targeted, while in the inpatient setting the instruction of parents in behavior management principles was not the main focus of the interventions.

As reported before (Appendix), ${ }^{10} 1916$ patients had finally been recruited into the EvAKuJ study and had been allocated to one of the two treatment settings. Comparison of baseline data had shown significant differ- ences between both patient groups: outpatients were slightly younger and had lower BMI-SDS values than inpatients (Appendix). ${ }^{10}$ Patient allocation and follow-up is summarized in Figure 1. The number of patients who did not complete the assigned treatment or who did not participate in the final assessment (or whose data were not transmitted to the study

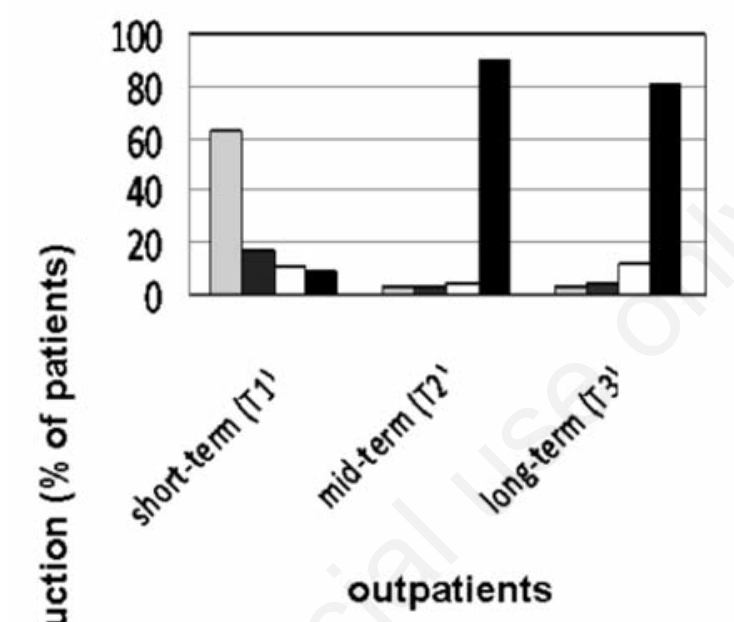

$\square$ successful (at least $-0,2$ but less than $-0.5 \mathrm{BMI}-\mathrm{SDS}$ )

good success (at least $-0,5$ BMI-SDS)

$\square$ not successful (less than0,2 BMI-SDS)

Drop-out (T1)/Loss-tofollow-up $(\mathrm{T} 2 ; \mathrm{T} 3)$

\section{inpatients}

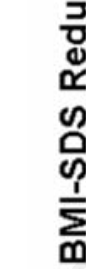

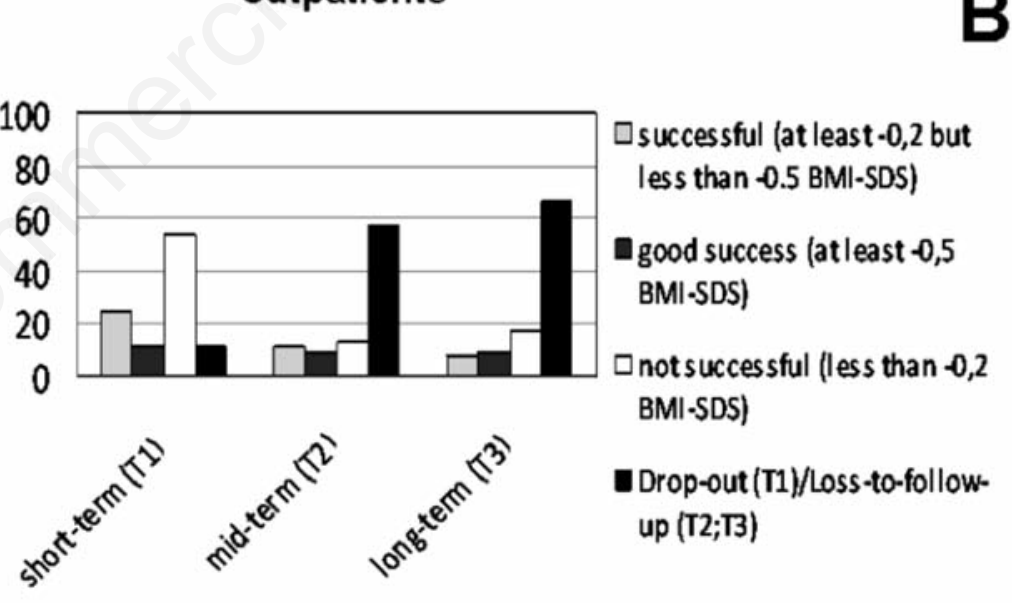

Figure 2. Short-term, mid-term, and long-term effects of obesity treatment on body-massindex standard deviation scores (BMI-SDS) in an inpatient setting (A) or an outpatient setting (B) as reported by the EvAKuJ Study Group (Appendix). Treatment was classified as successful if a patient reduced his or her BMI-SDS of at least -0.2 but less than -0.5 . BMI-SDS reduction of at least $\mathbf{- 0 . 5}$ was classified as good treatment success. Patients with BMI-SDS reduction of less than $\mathbf{- 0 . 2}$, no reduction at all, or an increase in BMI-SDS were grouped as not successful. The percentages of patients achieving these predefined changes in BMI-SDS were calculated using an Intention-to-Treat (ITT)-approach (i.e., counting patients who dropped out or were lost to follow-up as not successful). Short-term outcome data stem from measurements at the end of treatment (time point $T_{1}$ ) in 787 patients in the inpatient setting $(91 \%$ of 875 participants who were initially recruited) and 927 patients in the outpatient setting ( $89 \%$ of 1041 participants who were initially recruited). Mid-term outcome data stem from measurements one year after the end of treatment (time point $\mathrm{T} 2)$ in 88 patients in the inpatient setting (10\% of 875 participants who were initially recruited) and 452 patients in the outpatient setting (43\% of 1041 participants who were initially recruited). Long-term outcome data stem from measurements two years after the end of treatment (time point $\left.T_{3}\right)$ in 167 patients in the inpatient setting (19\% of 875 participants who were initially recruited) and 358 patients in the outpatient setting (34\% of 1041 participants who were initially recruited). 
center) at the end of the intervention $\left(\mathrm{T}_{1}\right)$ is labeled as drop-out. The number of patients who did not participate in the assessments one $\left(\mathrm{T}_{2}\right)$ or two $\left(\mathrm{T}_{3}\right)$ years after the end of the intervention - or whose data were not transmitted to the study center - is labeled as lossto-follow-up. At the end of the intervention there were no significant differences in age, gender distribution, and BMI-SDS at baseline $\left(\mathrm{T}_{0}\right.$ ) between patients who completed the intervention and those who dropped out, whereas patients who completed the intervention and participated in the follow-up assessments were slightly younger and had a significantly lower BMI-SDS at baseline than those lost to follow-up (Appendix). ${ }^{10}$

In the EvAKuJ Study, treatment success had been defined according to the national consensus statement as a reduction in the body-massindex standard deviation score (BMI-SDS) of at least $-0.2 ; 8 \operatorname{good}$ treatment success had been defined as a reduction in BMI-SDS of at least -0.5 . Treatment success rates in both settings at different time points have been published by the EvAKuJ Study Group ${ }^{10}$ (Appendix) both per protocol (pP; complete-case analysis) and as intention-to-treat (ITT) analysis (Appendix for definitions); these data are summarized in Figure 2. The following calculations had been performed separately for both treatment settings: i) short-term treatment success at the end of the intervention as the difference between the measurements at time points $\mathrm{T}_{0}$ and $\mathrm{T}_{1}$; ii) mid-term treatment success approximately one year after the end of the intervention as the difference between the measurements at time points $\mathrm{T}_{0}$ and $\mathrm{T}_{2}$; iii) long-term treatment success approximately two years after the end of the intervention as the difference between the measurements at time points $\mathrm{T}_{0}$ and $\mathrm{T}_{3}$.

All ITT calculations included patients who either dropped out (and did not complete the assigned intervention) or did not respond to the request for data communication and were thus lost to follow-up. All $\mathrm{pP}$ calculations included only completers, i.e., patients whose baseline and follow-up data at a defined time point were available. Due to a high loss to follow-up of patients at $\mathrm{T}_{2}$ and $\mathrm{T}_{3}$ the results of both calculations indicated different success rates: ${ }^{10}$ when analyzed $\mathrm{pP}, 60 \%$ of the patients were successful at the end of the intervention $\left(\mathrm{T}_{1}\right), 45 \%$ one year later $\left(\mathrm{T}_{2}\right)$, and $50 \%$ after two years $\left(\mathrm{T}_{3}\right)$. In the corresponding ITT analysis, $56 \%$ of the originally recruited 1.916 patients successfully completed the assigned intervention with a BMI-SDS reduction of at least -0.2 at $\mathrm{T}_{1}$, but only $13 \%$ and $14 \%$ continued to show this reduction one $\left(\mathrm{T}_{2}\right)$ or two $\left(\mathrm{T}_{3}\right)$ years later, respectively. In addition, the design of the EvAKuJ Study did not allow to document the spontaneous development of BMI-SDS over time in untreated overweight and obese children and adolescents - a fact which renders interpretation of study results by both ITT and pP analysis difficult.

In order to compensate for the large proportion of patients with missing data (drop-out and loss to follow-up at mid-term: $90 \%$ of the inpatients and $57 \%$ of the outpatients; drop-out and loss to follow-up at long-term: $81 \%$ of the inpatients and $66 \%$ of the outpatients) and to overcome the lack of an appropriate control group, we performed sensitivity analysis to describe possible health policy implications of the study results published by the EvAKuJ Study Group. To this purpose we calculated the odds ratio (OR) for successful treatment (BMISDS reduction of at least -0.2 and -0.5 , respectively) using the odds ratio calculator available at http://www.hutchon.net/ConfidORnulhypo. html, under the following assumptions: i) crude numbers of successful and unsuccessful participants at $\mathrm{T}_{1}, \mathrm{~T}_{2}$ and $\mathrm{T}_{3}$ were given separately for inpatients and outpatients and for the two modes of calculation (ITT and pP); ii)

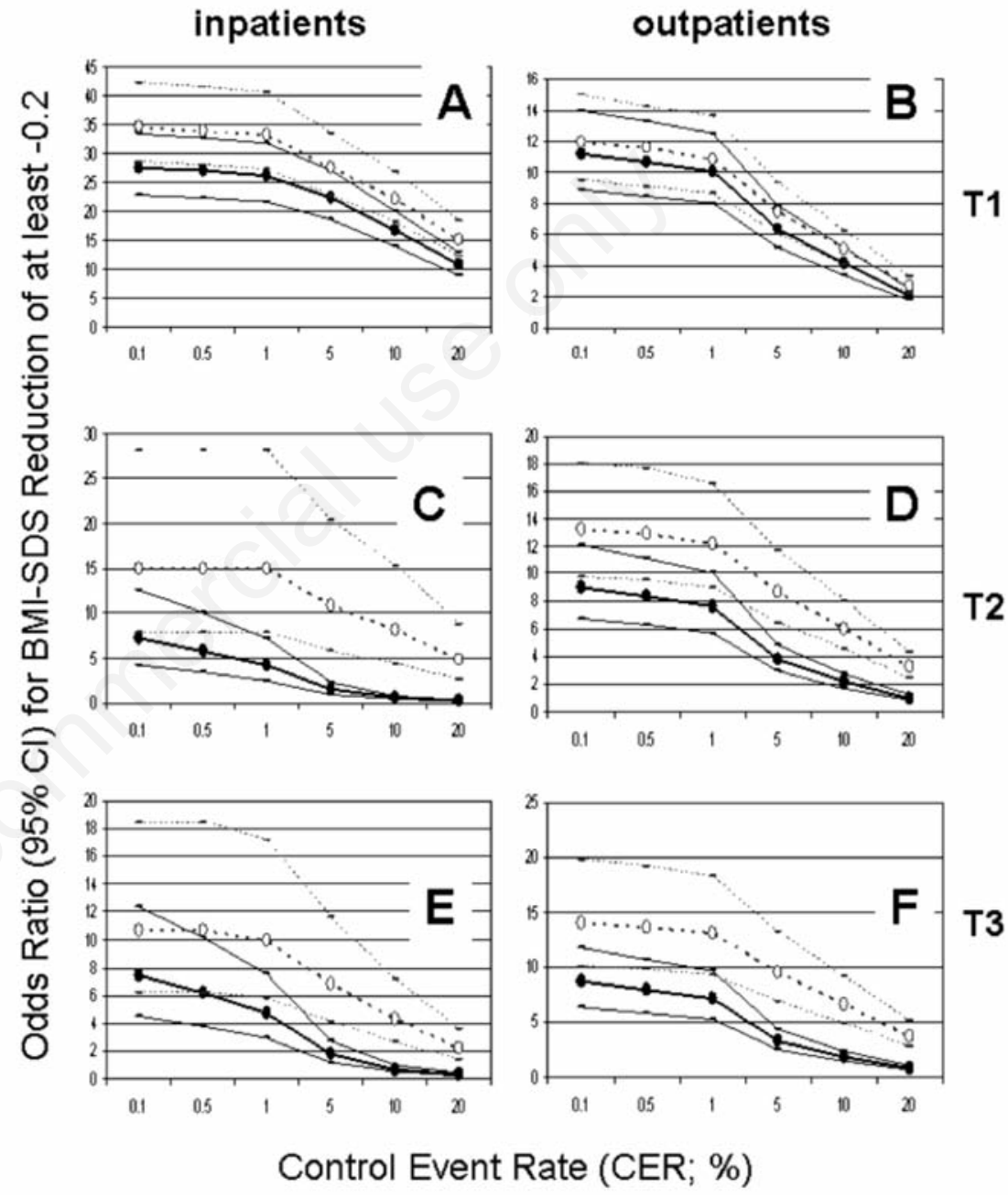

Figure 3. Odds ratios (OR) and upper and lower limits of $95 \%$ confidence intervals (CI) for successful obesity treatment [reduction of body-mass-index standard deviation scores (BMI-SDS) of at least -0.2] as a function of different categories of control event rates (CER) in a hypothetical group of untreated children and adolescents in the inpatient setting $(\mathrm{A}, \mathrm{C}$, and $\mathrm{E})$ and the outpatient setting $(\mathrm{B}, \mathrm{D}$, and F) of the EvAKuJ study. OR calculated using the Intention-to-Treat (ITT)-approach (i.e., counting patients who dropped out or were lost to follow-up as not successful) are indicated by black dots and solid lines, those calculated by the per protocol (pP)-approach (i.e., omitting patients who dropped out or were lost to follow-up from the calculation of treatment success rates) as grey circles and punctuated lines. $T_{1}, T_{2}$, and $T 3$ indicate different time points of follow-up: $T_{1}$ means approximately 6 weeks of follow-up in inpatients (A) and approximately 12 months of follow-up in outpatients (B), T1 indicates data obtained about one year after the end of the intervention in both treatment settings (C and D), and T3 stands for data obtained about two years after the end of the intervention in both treatment settings ( $\mathrm{E}$ and $\mathrm{F})$. 
a hypothetical control group with identical numbers of observations as the treatment groups was included; iii) in the hypothetical control group of untreated patients, a reduction of BMI-SDS of at least -0.2 or -0.5 was thought to occur spontaneously in $0.1 \%, 0.5 \%$, $1 \%, 5 \%, 10 \%$ and $20 \%$ at $\mathrm{T}_{1}, \mathrm{~T}_{2}$ and $\mathrm{T}_{3}$ (control event rate, CER).

This method allowed us to depict the OR for successful treatment together with its 95\% confidence interval - obtained from data published by the EvAKuJ Study Group - as a function of the CER in the hypothetical control group. Calculations were performed using Microsoft Excel 2003 for Windows.

The number of patients who had to be exposed to the intervention in order to see a reduction in BMI-SDS of at least -0.2 or -0.5 in one additional patient (number needed to benefit from treatment; NNT) was calculated as described, 18,19 using the same crude numbers of successful and unsuccessful participants and hypothetical control event rates for BMISDS reduction of at least -0.2 or -0.5 which were used for calculation of the $\mathrm{OR}$ for each time point and setting. The absolute risk of not reducing BMI-SDS was calculated for the treatment group and the respective hypothetical control group (for example, $20 \%$ for inpatients at $\mathrm{T}_{1}$, using the ITT approach for successful treatment $=\mathrm{R}_{\mathrm{T}} ; 95 \%$ for the hypothetical controls without treatment at a CER of 5\% = $\mathrm{R}_{\mathrm{w} / \mathrm{T}}$ ). The absolute risk reduction (ARR) due to treatment was calculated as the difference between $\mathrm{R}_{\mathrm{w} / \mathrm{T}}$ and $\mathrm{R}_{\mathrm{T}}$ (in our example: 75 ) and the NNT was calculated as 100/ARR (in our example: 1.33). The number needed to benefit from treatment is depicted as a function of the CER in the hypothetical control group. Calculations were performed using Microsoft Excel 2003 for Windows.

\section{Results}

Sensitivity analysis of published outcome data of the EvAKuJ study is summarized separately for inpatients and outpatients in Figure 3 (OR for BMI-SDS reduction of at least -0.2 , successful treatment), Figure 4 (OR for BMISDS reduction of at least -0.5 , good treatment success), and in Figure 5 (the respective NNT), each variable as a function of the CER in the hypothetical control group $(0.1 \%, 0.5 \%, 1 \%$, $5 \%, 10 \%$, and $20 \%$ ) at the different time points of follow-up $\left(\mathrm{T}_{1}, \mathrm{~T}_{2}\right.$ and $\left.\mathrm{T}_{3}\right)$.

At the end of the intervention $\left(\mathrm{T}_{1}\right)$, only few patients had dropped-out (88 inpatients and 114 outpatients) and ITT and pP analysis gave similar results in both treatment settings. OR for successful treatment are considerably higher in inpatients than in outpatients over the whole CER range (Figure 3A, B). OR for good treatment success, however, are nearly identical (Figure 4A, B), indicating that short inpatient treatment of overweight and obese children and adolescents is more effective than longer outpatient treatment in reducing BMI-SDS of at least -0.2 but does not lead to a higher proportion of patients with a BMI-SDS reduction of at least -0.5 (good treatment success). As shown in Figure 5, this translates into similar NNT for good treatment success both in inpatients (Figure 5A) and outpatients
(Figure 5B), while NNT for BMI-SDS reduction of at least -0.2 were lower in inpatients.

OR decrease and NNT increase with CER above $1 \%$ in the hypothetical control group. This reflects the interdependency between the calculated treatment success rate and the background risk in the population. 18 At the end of the intervention $\left(\mathrm{T}_{1}\right)$ the lower estimate of the $95 \%$ confidence interval (lower $95 \% \mathrm{CI}$ ) of the calculated OR for a BMI-SDS reduction of at least -0.5 (Figure 4A, B) falls below 1.0 (indicating absence of effectiveness) at a CER

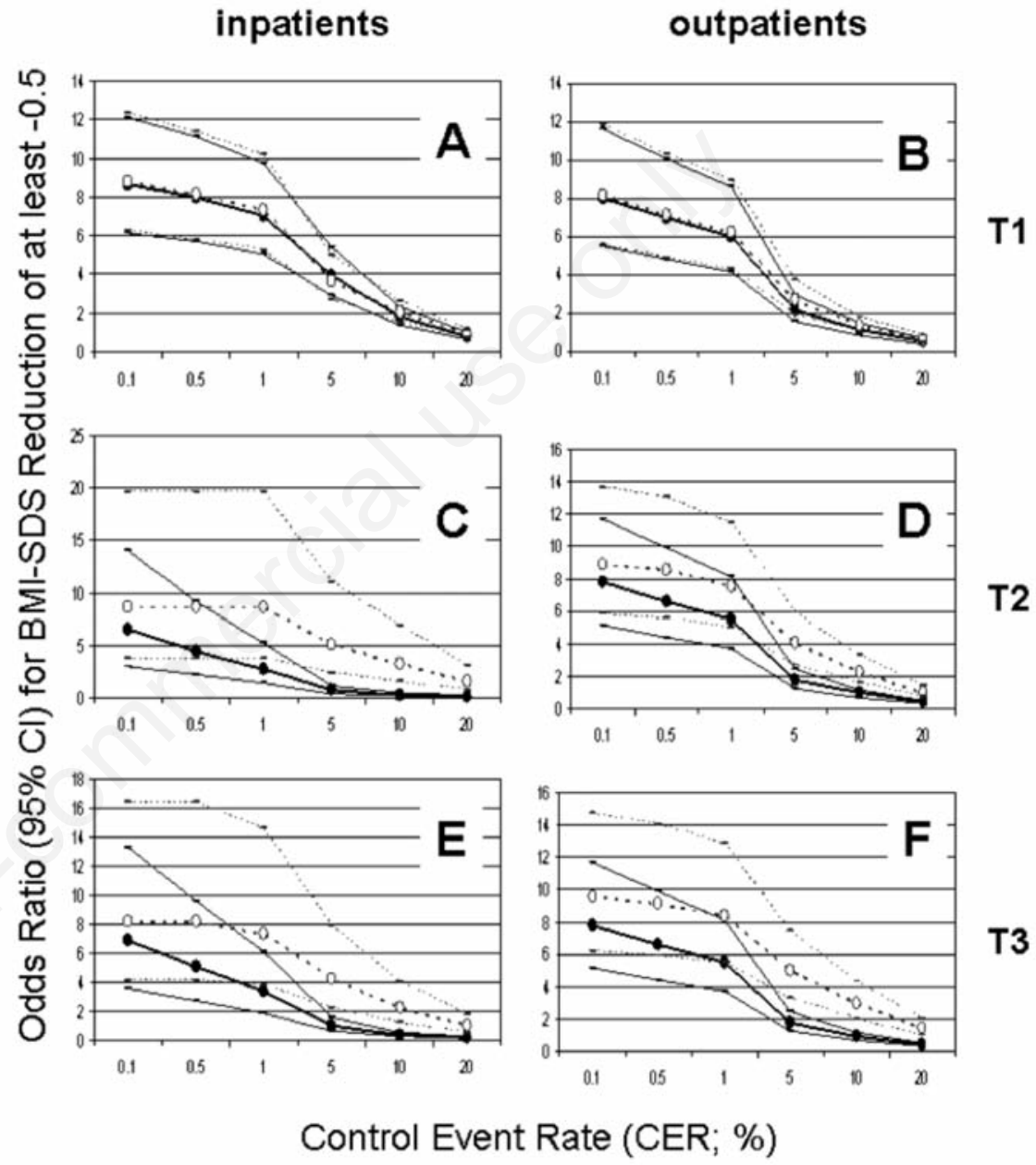

Figure 4. Odds ratios (OR) and upper and lower limits of $95 \%$ confidence intervals (CI) for good success in obesity treatment [reduction of body-mass-index standard deviation scores (BMI-SDS) of at least -0.5] as a function of different categories of control event rates (CER) in a hypothetical group of untreated children and adolescents in the inpatient setting (A, C, and E) and the outpatient setting (B, D, and F) of the EvAKuJ study. OR calculated using the Intention-to-Treat (ITT)-approach (i.e., counting patients who dropped out or were lost to follow-up as not successful) are indicated by black dots and solid lines, those calculated by the per protocol (pP)-approach (i.e., omitting patients who dropped out or were lost to follow-up from the calculation of treatment success rates) as grey circles and punctuated lines. $T_{1}, T_{2}$, and $T_{3}$ indicate different time points of follow-up: $T_{1}$ means approximately 6 weeks of follow-up in inpatients $(A)$ and approximately 12 months of follow-up in outpatients (B), T1 indicates data obtained about one year after the end of the intervention in both treatment settings $(C$ and $D)$, and $T_{3}$ stands for data obtained about two years after the end of the intervention in both treatment settings (E and $F)$. 
of $20 \%$ (which seems to be a rather unrealistic estimation of the CER in the real life situation). Under the assumption of a CER of $10 \%$ in inpatients (another unrealistic estimation in the real life situation of an intervention of 4 to 6 weeks' duration), the calculated NNT for successful treatment is still considerably low (ITT: 14, pP: 12; Figure 5A). In outpatients after approximately one year of treatment a CER of $10 \%$ would, however, cause a steep increase of the NNT for a BMI-SDS reduction of at least -0.2 (Figure $5 \mathrm{~B}$ ). The NNT resulting from ITT analysis $(\mathrm{n}=96)$ would be rather high and the NNT resulting from $\mathrm{pP}$ analysis $(n=41)$ would at least cast doubts on the effectiveness of such a treatment. 19

Sensitivity analysis of mid-term $\left(\mathrm{T}_{2}\right)$ and long-term $\left(\mathrm{T}_{3}\right)$ treatment success rates shows much larger differences between ITT and pP results than the short-term success rates. One and two years after the end of the interventions, the majority of patients was lost to follow-up or had dropped-out (Figure 1). While ITT analysis was based on 875 inpatients and 1041 outpatients, pP analysis relied on 88 inpatients and 452 outpatients at $\mathrm{T}_{2}$, and 167 inpatients and 358 outpatients at $\mathrm{T}_{3}$. Thus, both methods of data analysis give different results in sensitivity analysis, either in terms of OR for successful treatment or good treatment success (which are considerably lower using ITT analysis, Figures 3 and 4), or in terms of NNT (which are much higher in ITT analysis and increase steeply or even loose statistical significance at a CER above 1\%; Figure 5). Data analysis pP yields significant positive OR for successful treatment (lower 95\% CI above 1.0) over the whole CER range in both treatment settings (with the exception of BMI-SDS reduction of at least -0.5 in inpatients at $\mathrm{T}_{2}$, assuming a CER of 20\%; Figure 4C). ITT analysis at $\mathrm{T}_{2}$ reveals that the lower $95 \% \mathrm{CI}$ of the calculated OR for a BMI-SDS reduction of at least -0.2 (Figure 3C, D) decreases below 1.0 (indicating absence of effectiveness) at a CER of $5 \%$ in inpatients and of $20 \%$ in outpatients; for a BMI-SDS reduction of at least -0.5 they decrease below 1.0 at a CER of $5 \%$ in inpatients and of $10 \%$ in outpatients (Figure 4C, D). Similar results are obtained at $\mathrm{T}_{3}$ (Figures 3 and 4).

\section{Discussion}

The EvAKuJ study aimed at longitudinal observation of overweight and obese children and adolescents in Germany for at least two years after exposure either to a hospital-based (short, inpatient) treatment for less than 3 months, or to an extended ambulatory (long, outpatient) education and training program for approximately 12 months. Assessment of the patients directly after the end of the intervention suggested that especially inpatient treatment has the potential to effectively influence body weight leading to a clinically significant decrease in BMI-SDS. Yet, $11 \%$ of inpa-
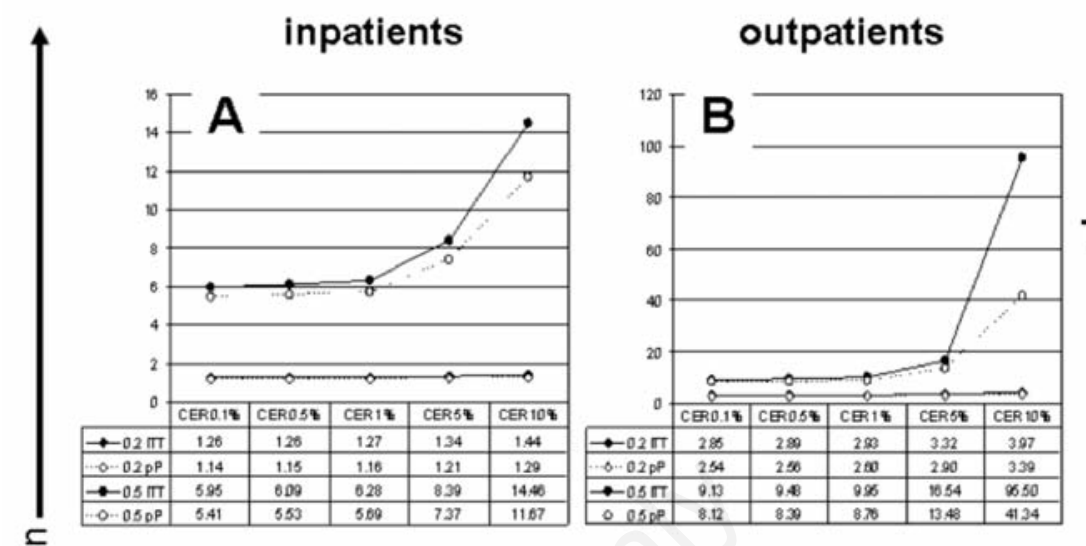

T1 tients and $54 \%$ of outpatients had either no relevant decrease or even an increase in BMISDS at the end of the intervention. 16

According to the EvAKuJ Study Group (Appendix), the study provides data on short term changes after lifestyle interventions for
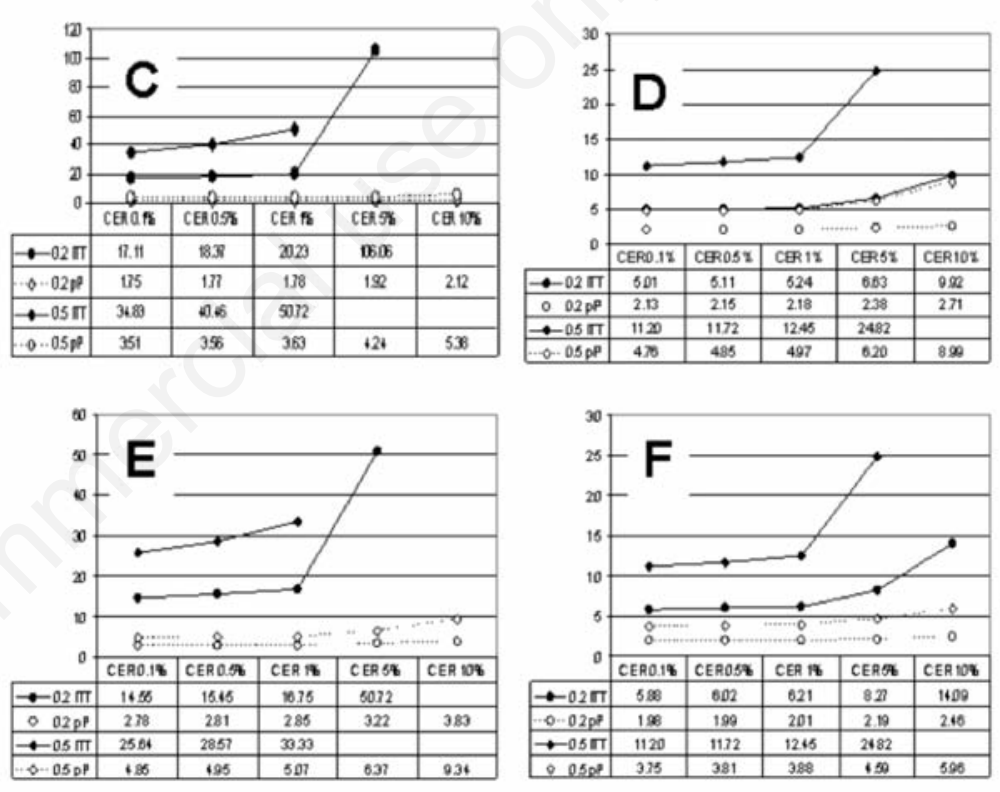

\section{Control Event Rate (CER; \%)}

Figure 5. The number needed to treat (NNT) in order to achieve a reduction of bodymass-index standard deviation scores [BMI-SDS] of at least $\mathbf{- 0 . 2}$ (dots and circles) or $\mathbf{- 0 . 5}$ (filled and empty diamonds) in one additional patient as a function of different categories of control event rates (CER) in a hypothetical group of untreated children and adolescents in the inpatient setting $(\mathrm{A}, \mathrm{C}$, and $\mathrm{E})$ and the outpatient setting $(\mathrm{B}, \mathrm{D}$, and F) of the EvAKuJ study. NNT calculated using the Intention-to-Treat (ITT)-approach (i.e., counting patients who dropped out or were lost to follow-up as not successful) are indicated by black dots or filled diamonds and solid lines, those calculated by the per protocol ( $\mathrm{pP}$ )-approach (i.e., omitting patients who dropped out or were lost to followup from the calculation of treatment success rates) as circles or empty diamonds and punctuated lines. The calculated NNT are shown in the tables below the $x$-axis of each plot. Empty cells in the tables indicate negative NNT, the corresponding symbols were omitted from the respective graph. $T_{1}, T_{2}$, and $T_{3}$ indicate different time points of follow-up: $T_{1}$ means approximately 6 weeks of follow-up in inpatients (A) and approximately 12 months of follow-up in outpatients (B), T2 indicates data obtained about one year after the end of the intervention in both treatment settings (C and D), and $T_{3}$ stands for data obtained about two years after the end of the intervention in both treatment settings (E and F). While NNT calculations using the ITT approach are based on 875 inpatients and 1041 outpatients, those based on the $\mathrm{pP}$ approach rely upon data from 787 inpatients and 927 outpatients at $T_{1}, 88$ inpatients and 452 outpatients at $T_{2}$, and 167 inpatients and 358 outpatients at $T_{3}$. 
overweight and obese children and adolescents in Germany under real-life conditions, but long term outcomes cannot be examined because of the high loss to follow-up. The EvAKuJ Study Group concluded that: i) single overweight or obese children or adolescents may benefit in terms of weight loss from current lifestyle interventions in both treatment settings; ii) single interventions, which differ considerably in the way they are organized and financed, may yield distinct patterns of outcome; iii) differences in results between single providers may be influenced by available resources (e.g., personnel, space, equipment), therapeutic processes (e.g., frequency and intensity of treatment sessions, skills and preferences of specific providers), and patient case mix (different age, degree of overweight, socioeconomic background of the family, and general motivation for treatment); iv) reliable conclusions regarding the importance of inpatient and outpatient interventions (or elements thereof) and the different treatment modalities outlined above cannot be drawn, due to the design of the EvAKuJ study.

A major problem is that $80 \%$ of the inpatients and $51 \%$ of the outpatients had dropped out or were lost to follow-up approximately one year after the end of the intervention. Another year later (i.e., two years after the end of the intervention), combined drop-out and loss to follow-up were $71 \%$ among inpatients and $61 \%$ among outpatients. Such a loss of information on individual outcomes after treatment seriously jeopardizes any conclusion, including ITT analysis of treatment success rates. The fact that missing data in clinical trials on overweight and obese subjects make analysis of trial results unreliable has been repeatedly discussed in the literature. ${ }^{20,21}$ Whenever possible, outcome data should be analyzed using the intention-to-treat (ITT) approach. The alternative statistical approach, using complete case or per protocol (pP) analysis, does not allow a scientifically sound conclusion from the EvAKuJ trial. Imputation of missing values in longitudinal data analysis is not an option either. Lack of data precludes researchers to complete empty cells in the data matrix by model-driven plausible values, because they cannot be predicted from empirical evidence. ${ }^{22}$ However, neither the assumptions of the model nor the mechanism leading to missing values can be verified in real life data or field study settings. All statistical approaches for post-hoc handling of missing data in clinical trials leave uncertainties about the scientific soundness of the conclusions. ${ }^{23}$ Given the very high rate of drop-out and loss-to-follow-up in both treatment settings, the EvAKuJ Study Group therefore refrained from using any model based imputation method for data analysis. Employing sensitivity analysis with standard methods of clinical epidemiology, ${ }^{18,19}$ we were able to assess the relevance of the treatment success rates previously reported by the EvAKuJ Study Group and to assess robustness of the conclusions drawn from the study despite high proportions of missing data at mid-term and long-term follow-up. Calculation of hypothetical OR in favor of successful treatment and the respective NNT in each setting also helped to compensate for the lack of an untreated control group in the design of the EvAKuJ study. Precise data on BMI-tracking in German youth are not available and the natural course of overweight and obesity in children and adolescents aged 8 to 16 years has not been assessed in detail. Indirect evidence for spontaneous resolution of overweight in children below 12 years of age is available in the literature. As reported by Oude Luttikhuis et al. 3 in untreated (waiting list) controls observed for 12 months, a mean \pm 1 SD reduction of BMI-SDS was seen in the range of $-0.15 \pm 0.47,{ }^{24}-0.18 \pm 0.28,25$ or $-0.1 \pm 0.3 .{ }^{26}$ Due to possible differences in the methodology of BMI-SDS calculations (the LMS method used by German research groups may yield lower age-related changes than other methods) ${ }^{15}$ it is difficult to estimate how many children may reduce BMI-SDS spontaneously by more than -0.2 in one year. In a randomized controlled trial of family-based behavioral treatment of childhood obesity in the United Kingdom it was not possible to identify a specific treatment effect after 6 months due to similar reductions of BMI-SDS both in the treatment group and in waiting-list controls. ${ }^{27}$ On the other side, Reinehr et al. ${ }^{28,29}$ showed that the lifestyle intervention Obeldicks light, based on 6 months of physical activity training, nutrition education, and behavior counseling for children and their parents, caused a significant mean reduction of BMI-SDS by $-0.26 \pm 0.22$ in a group of 34 overweight children (mean age, 11.6 \pm 1.6 years), while BMISDS increased by $0.05 \pm 0.19$ in the control group of 32 overweight children (mean age, $11.4 \pm 1.7$ years). Thus, it remains unclear which CER most accurately reflects spontaneous changes in BMI-SDS with age in German children and could therefore be used in sensitivity analysis for BMI-SDS reduction of at least -0.2 in this age group.

Nevertheless, the chance that older children and adolescents with higher BMI-SDS may spontaneously loose their overweight seems to be low. 30,31 Studies analyzed by Oude Luttikhuis et $a l .{ }^{3}$ in untreated (waiting list) controls above 12 years of age who were observed for 12 months revealed a mean $\pm 1 \mathrm{SD}$ change in BMI-SDS in the range of $0.0 \pm 0.1129$ or $-0.02 \pm 0.10 .32,33$ Assuming that BMI-SDS values are normally distributed, these data indicate that less than $5 \%$ of older children and adolescents may reduce BMI-SDS spontaneously by at least -0.2 in one year. Obese ado- lescents may remain obese without specific treatment and seem to benefit less from behavioural treatment. ${ }^{31}$ A significant proportion of overweight children and adolescents may spontaneously loose overweight when getting older (approximately 6\% in 6 months during the study of Reinehr et al.;28,29 unpublished data) or may increase BMI-SDS over time (approximately 22\% in 6 months during the study of Reinehr et al.;28,29 unpublished data).

The observation that younger and less overweight participants of the EvAKuJ study seem to benefit most from treatment in the short term (Appendix) may actually reflect both the spontaneous reduction of BMI-SDS which occurs over time in a subgroup of patients as well as better results of behavioural treatment using parents as agents of change in this age group. ${ }^{34}$ Especially pP analyses of these data are therefore difficult to interpret. Because the EvAKuJ study did not assess spontaneous changes of BMI-SDS in a control group of overweight and obese children and adolescents over time, the observed reductions in BMI-SDS (which were attributed to treatment effects in previous publications of the study group) may have occurred also due to age-specific physiologic development or by chance and may not completely be related to treatment.

One must admit weaknesses in study design (no control group), data analysis (the unit of analysis, i.e., the individual patient, differed from the unit of assignment, i.e., the health care provider - but no statistical method was used to account for a potential cluster effect; post-hoc change in the plan for data analysis switching from 6 clusters to 2 settings), as well as study performance (uncontrolled allocation of patients to the different clusters, no process control of the interventions, no screening whether the assigned intervention was delivered as planned, and - most importantly - a very high proportion of patients who were lost to follow-up). ${ }^{35}$ Yet, the methods used to collect data represent validated instruments, content and time course of the interventions have been described, and recruitment and characterization of patients in the treatment settings as well as drop-out and follow-up rates have been provided in a transparent manner (Appendix). ${ }^{10}$ Patients' baseline data have been extensively presented and the main outcome (BMI-SDS reduction at time points $\mathrm{T}_{1}$, $\mathrm{T}_{2}$, and $\mathrm{T}_{3}$ ) as well as the secondary outcomes have been calculated and reported using an intention-to-treat approach.

According to the model of stepwise implementation of complex health interventions into an existing health care system proposed by Campbell et al., ${ }^{9}$ an evaluation study should provide information on constant and variable elements of obesity treatment offered in Germany at that time (phase III). As outlined by Pfaff $e t$ al., 13 during such a study acceptabil- 
ity and feasibility of the intervention should be assessed, its design adapted, and the relevant outcome parameters and treatment in the control group should be determined - in order to prepare phase IV, the definitive randomized controlled effectiveness study. Should such a study be initiated now? The EvAKuJ study was initiated after analysis of the published evidence for effectiveness of interventions to treat obesity in children in the year 2004, 8,36 and after the available treatment options in Germany had been assessed in 2005.2 Given the limited evidence about success of treatment from the EvAKuJ study and taking into account the low reliability of its outcome data due to the known but accepted problems with study design and performance, the BZgA came to the conclusion that the necessary prerequisites for such a confirmative study are lacking - at least in the context of the German health care system. ${ }^{10}$ As Campbell et al. ${ }^{12}$ pointed out, the implementation process of complex health interventions into such a system has to be viewed as an iterative process of developments, appraisals, and re-appraisals in parallel. In retrospect, refraining from accurately organizing and appropriately financing longterm patient follow-up appears as the main pitfall in study design, being a probable cause of extensive loss to follow-up and impeding reliable long-term analysis of treatment success rates. Another aspect of the study which has been discussed in a previous publication of the EvAKuJ Study Group is the apparent heterogeneity of treatment results between single providers - at least in the outpatient setting (Appendix). ${ }^{10}$ One might speculate about the reasons of this variability and might try to find out how to learn from the best. However, before analyzing what is done differently in the most successful treatment centers, it is necessary to precisely identify those centers through benchmarking using outcome data which are adjusted for the existing baseline risk for treatment failure. Treatment success rates of different providers cannot be appropriately compared if confounders such as age and BMI-SDS before treatment are not taken into consideration.

Before a new study is planned, the following open questions should be answered: i) how is BMI-SDS in untreated overweight and obese children and adolescents tracking forward?; ii) how are effectiveness-related treatment processes put into clinical practice by each provider?; iii) how can appropriate indicators of long-term success of any treatment be ascertained and reliably assessed?

In the meantime, German pediatricians will continue to offer weight-reduction interventions to each child or adolescent with a relevant burden of disease due to obesity as outlined in the national consensus statement. ${ }^{8}$ Statutory Health Insurance Funds or Pension
Funds in Germany will continue to pay for inpatient rehabilitation of these children or offer outpatient education and training programs as outlined 8 years ago.8,36 However, there will be a closer look on how to individualize obesity treatment in German youth. Is it still reasonable to deliver a standardized complex intervention to every child or adolescent above a certain BMI-SDS limit? Both age and degree of overweight and obesity seem to influence results of lifestyle interventions in the long term,, 37 and the benefit of such a treatment seems to be rather limited especially in extremely obese adolescents. ${ }^{30}$ Certain elements of outpatient education and training programs may be sufficient for reaching individual treatment aims in younger children and less overweight patients. The interventions may become shorter or could be used in a modular way - which might help to increase motivation of the patients and their families. It should be investigated which treatment approaches yield the best outcome for certain patient subgroups and what characterizes effective delivery of care. Most importantly, documentation of treatment outcomes, especially of long-term results, should be required and budgeted appropriately at least in clinical studies - in order to avoid extensive loss to follow-up which is typical for obesity studies all over the world. 20

\section{Conclusions}

In sum: while outpatient treatment of overweight children and adolescents might fit the needs of many families, intensive inpatient treatment of obese children and adolescents could be seen as a powerful tool helping these patients to effectively reduce BMI-SDS in a relatively short period. This tool, however, has to be used carefully: the patients should be prepared for this treatment phase, and follow-up must be organized in the family and community setting in order to include all stakeholders. The discussion, how to implement such an even more complex health intervention into the existing health care system in Germany is ongoing. $38-40$

\section{References}

1. Kurth BM, Schaffrath Rosario A. Die Verbreitung von Übergewicht und Adipositas bei Kindern und Jugendlichen in Deutschland. Ergebnisse des bundesweiten Kinder- und Jugendgesundheitssurveys (KiGGS). Bundesgesundheitsblatt Gesundheits forschung Gesundheitsschutz 2007;50:
736-43.

2. Bundeszentrale für gesundheitliche Aufklärung (BZgA). Die Versorgung übergewichtiger und adipöser Kinder Quantität und Qualität von Hilfsangeboten im Zeitraum 2004-2005. Gesundheitsförderung konkret, Band 8. Köln: BZgA; 2007.

3. Oude Luttikhuis H, Baur L, Jansen H, et al. Interventions for treating obesity in children. Cochrane Database Syst Rev 2009;CD001872.

4. Whitlock EP, O'Connor EA, Williams SB, et al. Effectiveness of weight management interventions in children: a targeted systematic review for the USPSTF. Pediatrics 2010;125:e396-418.

5. Golan M. Parents as agents of change in childhood obesity--from research to practice. Int J Pediatr Obes 2006;1:66-76.

6. Konsensusgruppe Adipositasschulung (KgAS). Trainermanual Leichter, aktiver, gesünder. Interdisziplinäres Konzept für die Schulung übergewichtiger oder adipöser Kinder und Jugendlicher. 2nd ed. AID infodienst Verbraucherschutz, Ernährung, Landwirtschaft e.V. and Deutsche Gesellschaft für Ernährung (DGE) e.V. (eds.). Bonn: AID infodienst; 2007.

7. Bundesarbeitsgemeinschaft für Rehabilitation (BAR). Gemeinsames Rahmenkonzept der Gesetzlichen Krankenkassen und der Gesetzlichen Rentenversicherung für die Durchführung stationärer medizinischer Leistungen der Vorsorge und Rehabilitation für Kinder und Jugendlichen. Frankfurt am Main, 2008. Available from: http://www.bar-frankfurt. de/fileadmin/dateiliste/publikationen/arbe itsmaterialien/downloads/Gemeinsames_ Rahmenkonzept.pdf.

8. Böhler T, Wabitsch M, Winkler U. Consensus paper: patient training programmes for obese children and adolescents. In: Federal Centre for Health Education (BZgA). Quality criteria for programmes to prevent and treat overweight and obesity in children and adolescents. Methods of Health Promotion Vol. 4. Köln: BZgA; 2006. pp 37-55.

9. Campbell M, Fitzpatrick R, Haines A, et al. Framework for design and evaluation of complex interventions to improve health. Br Med J 2000;321:694-6.

10. Böhler T, Bengel J, Goldapp C, et al. Abschlussbericht im Rahmen des Qualitätssicherungsprozesses der BZgA zur Prävention und Therapie von Übergewicht bei Kindern und Jugendlichen. Köln: Bundeszentrale für gesundheitliche Aufklärung (BZgA); 2012. Available from: http://www.bzga-kinderuebergewicht.de/adipo_mtp/pdf/Bericht_EvA KuJ-Studie.pdf. 
11. von Elm E, Altman DG, Egger M, et al. The strengthening the reporting of observational studies in epidemiology (STROBE) statement: guidelines for reporting observational studies. PLoS Med 2007;4:e296.

12. Campbell NC, Murray E, Darbyshire J, et al. Designing and evaluating complex interventions to improve health care. $\mathrm{Br}$ Med J 2007;334:455-9.

13. Pfaff H, Albert US, Bornemann R, et al. Methoden für die organisationsbezogene Versorgungsforschung. Memorandum des Deutschen Netzwerks Versorgungsforschung. Gesundheitswesen 2009;71:77790.

14. Mühlhauser I, Lenz M, Meyer G. Entwicklung, Bewertung und Synthese von komplexen Interventionen - eine methodische Herausforderung. Z Evid Fortbild Qual Gesundh wesen (ZEFQ) 2011;105:751-61.

15. Kromeyer-Hauschild K, Wabitsch M, Kunze D, et al. Perzentile für den BodyMass-Index für das Kindes- und Jugendalter unter Heranziehung verschiedener deutscher Stichproben. Monatsschr Kinderheilkd 2001;149:807-18.

16. Perera R, Heneghan C, Yudkin P. A graphical method for depicting randomised trials of complex interventions. Br Med $\mathrm{J}$ 2007;334:127-9.

17. Wabitsch M, Kunze D. Leitlinien der Arbeitsgemeinschaft Adipositas im Kindes- und Jugendalter (AGA). Available from: http://www.dge.de/pdf/ll/LeitlinieA GA-Adipos itas - i m-Kindes-undJugendalter-2006.pdf.

18. Anlauf M. Assessing the efficacy of treatments using number needed to treat. Dtsch Arztebl 2006;103:A3254-8.

19. Sackett DL, Straus SE, Richardson WS, et al, eds. Evidence-based medicine. 2nd ed. Edinburgh: Churchill Livingstone; 2000. pp 113-117.

20. Ware $\mathrm{JH}$. Interpreting incomplete data in studies of diet and weight loss. N Engl J Med 2003;348:2136-7.

21. Little RJ, D'Agostino R, Cohen ML, et al. The prevention and treatment of missing data in clinical trials. N Engl $\mathrm{J}$ Med 2012;367:1355-60.

22. Yang X, Li J, Shoptaw S. Imputation-based strategies for clinical trial longitudinal data with nonignorable missing values. Stat Med 2008;27:2826-49.

23. DeSouza CM, Legedza AT, Sankoh AJ. An overview of practical approaches for handling missing data in clinical trials. $\mathbf{J}$ Biopharm Stat 2009;19:1055-73.

24. Golley RK, Magarey AM, Baur LA, et al. Twelve-month effectiveness of a parentled, family-focused weight management program for prepubertal children: a randomized, controlled trial. Pediatrics 2007;119:517-25.

25. Hughes AR, Stewart L, Chapple J, et al. Randomized, controlled trial of a bestpractice individualized behavioral program for treatment of childhood overweight: Scottish Childhood Overweight Treatment Trial (SCOTT). Pediatrics 2008;121:e539-46.

26. Kalavainen MP, Korppi MO, Nuutinen OM. Clinical efficacy of group-based treatment for childhood obesity compared with routinely given individual counseling. Int $\mathrm{J}$ Obes (Lond) 2007;31:1500-8.

27. Croker H, Viner RM, Nicholls D, et al. Family-based behavioural treatment of childhood obesity in a UK National Health Service setting: randomised controlled trial. Int J Obes (Lond) 2012;36:16-26.

28. Reinehr T, Schaefer A, Winkel K, et al. An effective lifestyle intervention in overweight children: findings from a randomized controlled trial on Obeldicks light. Clin Nutr 2010;29:331-6.

29. Schaefer A, Winkel K, Finne E, et al. An effective lifestyle intervention in overweight children: one-year follow-up after the randomized controlled trial on Obeldicks light. Clin Nutr 2011;30:629-33.

30. Danielsson P, Kowalski J, Ekblom Ö, Marcus C. Response of severely obese children and adolescents to behavioral treatment. Arch Pediatr Adolesc Med 2012;166: 1103-8.

31. Whitaker RC, Wright JA, Pepe MS, et al.
Predicting obesity in young adulthood from childhood and parental obesity. N Engl J Med 1997;337:869-73.

32. Savoye M, Shaw M, Dziura J, et al. Effects of a weight management program on body composition and metabolic parameters in overweight children: a randomized controlled trial. JAMA 2007;297:2697-704.

33. Williamson DA, Martin PD, White MA, et al. Efficacy of an internet-based behavioral weight loss program for overweight adolescent African-American girls. EWD 2005;10:193-203.

34. Golan M. Parents as agents of change in childhood obesity - from research to practice. Int J Pediatr Obes 2006;1:66-76.

35. Oakley A, Strange V, Bonell C, et al. Process evaluation in randomised controlled trials of complex interventions. $\mathrm{Br}$ Med J 2006;332:413-6.

36. Böhler T, Alex C, Becker $\mathrm{E}$, et al. Qualitätskriterien für ambulante Schulungsprogramme für übergewichtige und adipöse Kinder und Jugendliche. Gesundheitswesen 2004;66:748-53.

37. Reinehr T. Effectiveness of lifestyle intervention in overweight children. Proc Nutr Soc 2011;70:494-505.

38. Van Egmond-Fröhlich A, Brauer W, Goldschmidt H, et al. Effects of a programme for structured outpatient followup care after inpatient rehabilitation of obese children and adolescents -- a multicentre, randomized study. Die Rehabilitation (Stuttgart) 2006;45:40-51.

39. Stachow R, Sievers-Böckel B, Büssenschütt A, et al. Evidenzbasiertes Konzept zur Nachsorge für Kinder und Jugendliche mit Adipositas nach ambulanter oder stationärer Rehabilitation. Prävention Rehab 2012;24:110-20.

40. Adam S, Westenhöfer J, Rudolphi B, Kraaibeek HK. Effects of a Combined Inpatient-Outpatient Treatment of Obese Children and Adolescents. Obes Facts 2009;2:286-93. 